\title{
Criminologie
}

\section{Troubles de la personnalité et viol : implications théoriques et cliniques}

\author{
Jean Proulx, Jocelyn Aubut, Lise Perron et André McKibben
}

Volume 27, numéro 2, 1994

Sexe et criminalité

URI : https://id.erudit.org/iderudit/017354ar

DOI : https://doi.org/10.7202/017354ar

Aller au sommaire du numéro

\section{Éditeur(s)}

Les Presses de l'Université de Montréal

ISSN

0316-0041 (imprimé)

1492-1367 (numérique)

Découvrir la revue

Citer cet article

Proulx, J., Aubut, J., Perron, L. \& McKibben, A. (1994). Troubles de la personnalité et viol : implications théoriques et cliniques. Criminologie, 27(2), 33-53. https://doi.org/10.7202/017354ar
Résumé de l'article

During the past 30 years, behavioral and cognitive-behavioral theories of rape have evolved considerably. The influence of etio-logic factors related to personality, however, is limited to antisocial traits. The aim of the current study was, therefore, to investigate the presence of personality disorders in rapists. Forty-nine incarcerated rapists answered a French translation of the Millon Clinical Multiaxial Inventory questionnaire. On the basis of the Avery-Clark and Laws criteria (1983), 31 rapists were classified as less physically violent and 18 as more physically violent. Among the less physically violent rapists, we encountered most frequently avoidant, dependant, passive-aggressive and schizoid personality disorders. Among the more physically violent rapists, an antisocial personality disorder was predominant. The implications of these results concerning rape theories are being discussed. 
During the past 30 years, behavioral and cognitive-behavioral theories of rape have evolved considerably. The influence of etiologic factors related to personality, however, is limited to antisocial traits. The aim of the current study was, therefore, to investigate the presence of personality disorders in rapists. Fortynine incarcerated rapists answered a French translation of the Millon Clinical Multiaxial Inventory questionnaire. On the basis of the Avery-Clark and Laws criteria (1983), 31 rapists were classified as less physically violent and 18 as more physically violent. Among the less physically violent rapists, we encountered most frequently avoidant, dependant, passive-aggressive and schizoid personality disorders. Among the more physically violent rapists, an antisocial personality disorder was predominant. The implications of these results concerning rape theories are being discussed.

Au cours des trente dernières années, les théories comportementales et cognitivo-comportementales du viol ont évolué considérablement. Ainsi. selon Bond et Evans (1967), le viol serait la résultante d'un seul facteur. soit une préférence sexuelle pour le viol. Une décennie plus tard, Barlow et Abel (1976) considèrent que le viol serait la conséquence, non seulement d'une préférence sexuelle pour le viol, mais également de l'absence d'une préférence pour des contacts sexuels entre un homme et une femme adulte consentante, ainsi que de déficits quant aux habiletés sociales nécessaires pour accéder à une telle partenaire. Durant les années 1980, les modẻles proposés incluent les facteurs sexuels des modèles précédents, mais également des facteurs non-sexuels. Parmi ceux-ci, on retrouve les habiletés sociales. les habiletés de résolution de problèmes, les habiletés professionnelles ainsi que des distorsions cognitives relatives aux femmes (Marshall, Earls, Segal et Darke, 1983). Ces facteurs non-sexuels constituent des obstacles à un fonctionnement satistaisant sur les plans interpersonnel et professionnel, ce qui engendre du stress. Or ce stress peut précipiter la perpétration d'un viol chez un individu présentant une préférence sexuelle

1. Université de Montréal, École de criminologie, Centre international de criminologie comparée, 3150 Jean-Brillant, C. P. 6128, Succursale Centre-ville, Montréal (Québec) Canada H3C 3J7

2. Institut Philippe-Pinel de Montréal, 10905, boul. Henri-Bourassa est, Montréal (Québec) Canada H IC IHI 
pour le viol. Ainsi, malgré la complexité croissante des théories du viol, les caractéristiques de la personnalité ne tont pas partie des facteurs étiologiques considerés dans celles-ci.

Les modèles du viol proposés récemment sont plus explicites sur cette question. Ainsi, Marshall et Barbaree (1990) soutiennent que les caractéristiques de la personnalité antisociale constituent à la fois un facteur causal direct et un facteur facilitant, et ce en ce qui a trait aux comportements sexuels coercitifs vis-à-vis les femmes. En effet. les hommes présentant une personnalité antisociale dans leurs rapports interpersonnels manifestent des sentiments d'hostilité, un intérêt exclusif pour leurs besoins immédiats de même qu'un manque de sensibilité au vécu de l'autre. En conséquence. lorsque ce type de mode relationnel se manifeste dans la sphère sexuelle, il constitue une cause directe de comportements sexuels coercitifs. À un autre niveau, lorsqu'un individu présente une personnalité antisociale, il est plus perméable aux influences culturelles (médias, pairs) qui prônent la domination sexuelle et non sexuelle de la femme par l'homme. Or l'adhésion à ce type d'attitudes constitue l'une des causes directes du viol. En conséquence, les caractéristiques de la personnalité antisociale favorisent indirectement la propension au viol. Par-dela ces facteurs intra-psychiques et culturels, Marshall et Barbaree mentionnent également d'autres types de causes au viol, soit les facteurs biologiques, neurologiques et endocriniens, et les facteurs transitoires (ex. : alcool, drogue. colerre contre une femme).

Malgré l'intérêt que présente le modèle du viol proposé par Marshall et Barbaree, certains de ses aspects sont critiquables. Tout d'abord, l'influence des facteurs étiologiques reliés à la personnalité y est limitée à celle des traits antisociaux. En outre, leur modèle du viol ne rend pas compte de la diversité des types de violeurs, laquelle est pourtant bien documentée dans les études typologiques (Knight et Prentky, 1990; Knight, Rosenberg et Schneider, 1985) et phallométriques (Proulx. Aubut, McKibben et Côté. 1994).

À la différence du modèle proposé par Marshall et Barbaree, celui de Hall et Hirschman (1991) comporte une explication de l'hétérogénété des violeurs. Ainsi, selon leur modèle quadripartite, le viol résulterait de l'interaction de quatre types de facteurs, soit : 1) une excitation sexuelle pour le viol; 2) des distorsions cognitives qui justifient le viol : 3) de l'hostilité face aux femmes; 4) une personnalité antisociale. De plus, bien qu'ils considèrent que ces quatre facteurs sont présents chez tous les violeurs, il n'en existerait qu'un seul pour chacun d'eux qui serait un précurseur primaire. Ainsi. ce serait la nature de ce précurseur qui déterminerait le type de violeur. Bien que leur modèle comporte une explication de l'hétérogénéité des violeurs, l'influence des facteurs liés à la personnalité est limitée aux seules caractéristiques de la personnalité antisociale. 
Alors que la théorie du viol de Marshall et Barbaree ainsi que celle de Hall et Hirschman furent élaborées suite à des recherches effectués auprès de violeurs judiciarisés, celle de Malamuth et de ses collègues (Malamuth, 1986; Malamuth. Heavey et Linz, 1993; Malamuth. Sockloskie, Koss et Tanaka, 1991) le fut à partir de l'étude de sujets non judiciarisés provenant de collèges américains. Selon ces chercheurs, les comportements sexuels coercitifs à l'égard des femmes résultent de l'interaction de facteurs appartenant à l'une ou l'autre des dimensions que sont les attitudes masculines hostiles et la promiscuité sexuelle. Par cette dernière, ils entendent la précocité des premiers contacts sexuels ainsi que la multiplicité des partenaires sexuelles. Quant aux attitudes masculines hostiles, elles comportent trois volets, soit: 1) une tendance générale à la coercition: 2) de l'hostilité envers les femmes ; 3 ) des croyances qui justifient le viol. Dans cette théorie du viol, aucun type de personnalite n'est mentionné explicitement comme facteur étiologique. Toutefois, Malamuth et al. (1993) soulignent que la tendance générale à la coercition correspond à la dimension négative du narcissisme, c'est-à-dire à une propension à manifester un intêrêt exclusif pour ses besoins. associée à un manque de considération pour les conséquences des actes posés sur autrui. Donc, cette fois-ci, la dimension personnalité est limitée à une seule catégorie, soit le narcissisme. De plus, la théorie du viol de Malamuth et de ses collègues ne comporte pas d'éléments concernant la diversité des types de violeurs.

Somme toute, les théories du viol développées au cours des trois dernières décennies sont d'une complexité croissante. Cependant, plusieurs questions restent toujours sans réponse. En effet, la dimension personnalité est limitée aux personnalités antisociales et narcissiques. De plus, la diversité des violeurs est, dans bien des cas, escamotée au profit d'un modèle universel des causes du viol. Concernant ces deux derniers points, les études psychométriques offrent des avenues à explorer.

Plusieurs études psychométriques, basées sur le Minnesota Multiphasic Personality Inventory (MMPI), furent réalisées afin de clarifier les caractéristiques de personnalité des violeurs (Hall, Graham et Shepherd, 1991 ; Kalichman 1990, 1991 ; Kalichman, Szymanowski, Mc Kee, Taylor \& Craig, 1989 ; Levin et Stava. 1987). Les études dans lesquelles les caractéristiques de personnalité de violeurs furent comparées à celles de pédophiles ainsi qu'à celles de criminels non délinquants sexuels ne comportent pas de différence significative (Anderson, Kunce et Rich, 1979; Armentrout et Hauer, 1978; Kalichman, 1991; Karacan, Williams. Guerrero, Scales, Thornby et Hursch, 1974 ; Panton, 1958, 1978; Quinsey, Arnold et Pruesse, 1980). Il ne semble donc pas y avoir de profil de personnalité qui soit spécifique aux violeurs. Cependant, chez les délinquants sexuels dont les délits comportent un niveau plus élevé de violence physique, on retrouve un profil de personnalité distinct qui se caractérise par des élévations 
importantes à l'échelle de schizophrénie (Armentrout et Hauer, 1978 ; Panton. 1978) ainsi qu'aux échelles de paranoïa et d'hypomanie (Panton. 1978). Ces derniers résultats suggèrent donc qu'il y a des sous-types de délinquants sexuels qui se distinguent les uns des autres quant aux caractéristiques de la personnalité de même qu'en fonction du niveau de violence physique utilisée lors du délit. Ces résultats concordent avec ceux des typologies des délinquants sexuels dans lesquelles une place importante est accordée à cette dimension qu'est le niveau de violence physique (Knight et Prentky, 1990; Knight, Rosenberg et Schneider, 1985).

Hall, Graham et Shepherd (1991) ont abordé les caractéristiques de personnalité des délinquants sexuels à partir d'analyses factorielles portant sur les résultats au MMPI des sujets de leur échantillon constitué de violeurs et de pédophiles. Ces analyses ont permis d'identifier deux sous-types d'agresseurs sexuels. Ceux d'un premier sous-type ont présenté une forte élévation a l'échelle de psychopathie. Ces sujets se caractérisent donc par une faible tolérance à la frustration et par une tendance à avoir recours à la violence pour résoudre les conflits interpersonnels. Quant à ceux du second sous-type, ils ont manifesté des élévations marquées aux échelles de schizophrénie, de dépression et de psychopathie. Ces délinquants sexuels ont une faible estime de soi et des besoins de dépendance importants. Alors que cette recherche fut réalisée avec un groupe hétérogène de délinquants sexuels, Kalichman (Kalichman, 1990; Kalichman et al. 1989) en réalisa deux avec des échantillons composés uniquement de violeurs.

Il effectua des analyses de typologie sur des résultats au MMPI afin d'identifier des sous-types de violeurs. Lors de la première étude effectuée en 1989, il identifia cinq sous-types de violeurs. Les violeurs d'un premier sous-type n'ont présenté aucune élévation significative aux échelles du MMPI, alors que ceux du second sous-type ont obtenu une élévation significative à l'échelle de psychopathie. Les violeurs de ces deux sous-types ont habituellement commis leur crime sexuel lors de la perpétration d'un vol. En ce qui concerne les violeurs du troisième sous-type, leur profil de personnalité comporte des élévations importantes pour les échelles de dépression, de paranoïa, de schizophrénie et de psychopathie. Ces violeurs se caractérisent par une grande instabilité affective et une dominance de la colère dans leur répertoire de réactions émotionnelles. Leurs viols sont habituellement non prémédités et comportent un niveau de violence physique élevé. Pour ce qui est des violeurs du quatrième sous-type, des élévations significatives furent obtenues pour les échelles de psychopathie, de schizophrénie et de manie. Les violeurs de ce sous-type présentent une faible estime de soi et une difficulté a établir des relations intimes. Lors de leurs délits sexuels, le niveau de violence physique est généralement minimal et vise à obtenir la soumission de la victime. Finalement, les violeurs du cinquième sous-type ont présenté des élévations marquées pour la quasi- 
totalité des échelles du MMPI. Ce type de violeurs se caractérise par une fantasmatique de viol sadique envahissante et des perturbations affectives importantes. Lors des délits. le niveau de violence physique est élevé et souvent ritualisé. Les résultats de cette étude furent reproduits dans une seconde étude que Kalichman réalisa en 1990 avec un autre échantillon de violeurs.

Les études effectuées à partir du MMPI, que ce soit avec un groupe hétérogène d'agresseurs sexuels ou avec un groupe composé de violeurs uniquement, indiquent toute une diversité dans la psychopathologie rencontrée chez ces sujets. Toutefois, bien que le MMPI constitue un instrument de mesure valable en ce qui a trait à la psychopathologie. il n'est pas une mesure appropriée de la personnalité et encore moins des troubles de la personnalité (Levin et Stava, 1987 : Butcher et Tellegen, 1978 ; Kalichman. 1991 ; Millon, 1983). En effet. ce test qui fut développé durant les années 1940 ne rend pas compte de l'évolution importante des théories de la personnalité normale et pathologique réalisée depuis lors. C'est pourquoi certains chercheurs ont plutôt suggéré d'utiliser le Millon Clinical Multiaxial Inventory (MCMI) (Millon, 1983) afin d'évaluer les troubles de la personnalité d'agresseurs sexuels (Bard et Knight, 1987; Langevin, Lang, Reynolds, Wright, Garrels, Marchese. Handy, Pugh et Frenzel, 1988). Cette suggestion n'est pas surprenante, car ce questionnaire, à la différence du MMPI, fut développé spécifiquement pour évaluer les troubles de la personnalité. De plus, les échelles du MCMI correspondent aux catégories de troubles de la personnalité du DSM-III-R (APA, 1987). Or celui-ci constitue actuellement la nosologie psychiatrique la plus largement acceptée.

Bard et Knight (1987) ont administré le MCMI à des délinquants sexuels, violeurs ou pédophiles. Ils ont ensuite effectué des analyses de typologie, lesquelles indiquent la présence de quatre sous-types de délinquants sexuels dans leur échantillon. Ceux d'un premier sous-type ont présenté des élévations significatives aux échelles mesurant les troubles de la personnalité évitante, dépendante et schizoïde (groupe $\mathrm{C}$, anxieux). Ces délinquants sexuels se caractérisent par une faible estime de soi et leurs délits comportent un niveau de violence physique minimal. Les délinquants sexuels du second sous-type, majoritairement des violeurs, ont montré des élévations significatives aux échelles évaluant les troubles de la personnalité narcissique, antisociale et histrionique (groupe B. dramatique). Ces délinquants sexuels, particulièrement hostiles dans leurs rapports interpersonnels, ont perpétré des délits qui comportent un niveau de violence physique élevé. Quant aux délinquants sexuels du troisième soustype, des élévations significatives furent obtenues pour les échelles mesurant les troubles de la personnalité antisociale et passive-agressive. Ils présentent des troubles liés à l'utilisation de substances psycho-actives. Leur criminalité est généralement contre la personne plutôt qu'économique. 
Finalement. les délinquants sexuels du quatrième sous-type ne montrent aucune élévation significative aux échelles de troubles de la personnalité du MCMI.

Une autre étude sur les troubles de la personnalité chez des délinquants sexuels fut réalisée par Langevin et al. (1988), à l'aide du MCMI. Leur étude porta sur quatre groupes de délinquants sexuels (violeurs. pédophiles homosexuels, pédophiles hétérosexuels. incestueux), ainsi que sur un groupe témoin. Les résultats moyens aux échelles du MCMI ne diffèrent pas pour les groupes de délinquants sexuels. Toutefois, ils ditfèrent de ceux du groupe témoin. En effet, les sujets de ce dernier groupe ont présenté des élévations moyennes plus importantes que celles des délinquants sexuels et ce pour les échelles des troubles de la personnalité obsessionnelle-compulsive et narcissique. Cependant, des résultats inverses furent notés pour les échelles des troubles de la personnalité évitante, dépendante, passive-agressive et schizoïde. Finalement, les résultats de cette étude indiquent que $90,2 \%$ des délinquants sexuels présentent au moins une élévation significative à une échelle de troubles de la personnalité. Ce dernier résultat met en lumière l'importance d'analyser minutieusement le rôle des troubles de la personnalité dans la genèse et le maintien des comportements sexuels déviants.

Ainsi, les études psychométriques, qu'elles aient été réalisées avec le MMPI ou avec le MCMI, indiquent une diversité des caractéristiques de personnalité des violeurs. En conséquence, ces résultats soulignent une faiblesse des théories du viol, lesquelles ne font référence qu'au trouble de la personnalité antisociale. Il est vrai que ce type de personnalité apparaît dans au moins une des catégories de chacune des typologies découlant des études psychométriques. Cependant, d'autres troubles de la personnalité sont également présents dans les autres catégories de ces typologies. Toutefois, afï de clarifier cette question, des études complémentaires sont nécessaires. En effet, alors que les études de violeurs effectuées à l'aide du MMPI posent problème à cause de la nature même de ce test, celles réalisées avec le MCMI sont problématiques en raison de l'hétérogénéité des échantillons étudiés. En conséquence, le but premier de la présente étude est d'évaluer, à l'aide du MCMI, les troubles de la personnalité de violeurs exclusivement. De plus, nous voulons vérifier s’il existe un lien entre le degré de violence physique lors du viol d'une femme adulte et le type de troubles de la personnalité identifié chez l'auteur d'un tel acte. Cette mise en relation nous apparaît particulièrement pertinente, car le niveau de violence physique constitue une dimension phénoménologique centrale dans la caractérisation des sous-types de violeurs, et ce tant dans les typologies cliniques (Knight et Prentky, 1990) que dans les typologies psychométriques (Kalichman, 1990; Kalichman et al. 1989). 


\section{MÉTHODOLOGIE}

\section{Sujets et procédures}

Lors de la présente étude. 55 violeurs furent évalués. Ceux-ci avaient tous été reconnus coupables d'au moins une agression sexuelle contre une femme adulte. De plus. leur dossier ne devait pas comporter d'antécédents d'agressions sexuelles contre des mineurs. Ces violeurs étaient âgés en moyenne de 32,6 ans (écart type : 6,4 ans) et ils purgaient en moyenne une sentence de 5.2 ans (écart type : 2,8 ans). Pour chacun d'eux, les données furent recueillies au cours de leur premier mois d'implication dans un programme de traitement pour agresseurs sexuels. Ce programme est en application à l'Institut Philippe-Pinel de Montréal, un hôpital psychiatrique à sécurité moyenne.

Pour chaque sujet, le niveau de violence physique présent lors de leur dernière agression sexuelle. fut évalué par deux juges à partir de leur dossier criminologique, lequel contient entre autres la déclaration de la victime. Ces deux juges ont utilisé les critères d'Avery-Clark et Laws (1983) pour évaluer le niveau de violence physique. Ainsi les violeurs classés peu violents physiquement devaient répondre aux critères suivants : 1) ne pas avoir utilisé plus de violence physique qu'il n'en fallait pour soumettre la victime: 2 ) avoir utilisé principalement des tactiques psychologiques de persuasion; 3) avoir posé principalement des actes sexuels, génitaux et prégénitaux, et ce par opposition à des actes essentiellement agressifs ; 4) une fois la victime soumise, avoir cessé d'utiliser la coercition physique lorsque celle-ci exprimait de la douleur, de l'inconfort ou du déplaisir. Quant aux violeurs classés très violents physiquement. ils répondaient aux critères suivants : 1) avoir utilisé plus de violence physique. qu'il n'en fallait pour contraindre la victime : 2) avoir principalement utilisé des tactiques de persuasion physiquement violentes ; 3 ) avoir posé des actes sexuels et non sexuels répondant principalement à des pulsions agressives plutôt que sexuelles; 4) une fois la victime soumise, avoir continué à exercer de la coercition physique, même si celle-ci exprimait de la douleur, de l'inconfort et du déplaisir. Puisque six sujets furent classés différemment par les deux juges. ils furent exclus de la présente étude. Ainsi, notre échantillon final est constitué de 31 violeurs classés peu violents physiquement et de 18 violeurs classés très violents physiquement.

\section{Instrument de mesure}

Les troubles de la personnalité des sujets furent évalués à partir du Millon Clinical Multiaxial Inventory (MCMI) (Millon, 1983). Plus spécifiquement, on a utilisé une version française, validée auprès d'un échantillon composé de Québécois francophones (D’Elia, 1988). Ce test objectif consiste en 175 questions auxquelles les sujets évalués devaient 
répondre vrai ou faux. Suite à la première étape de la procédure de compilation, on obtient une quantification brute pour chacun des onze troubles de la personnalité du DSM-III-R, soit : la personnalité schizoïde, évitante, đépendante, histrionique, narcissique, antisociale, obsessionnelle-compulsive, passive-agressive, schizotypique, état-limite, et paranoïde. Cette première étape fournit également des données brutes qui concernent neuf troubles de l'axe I du DSM-III-R (ex. : dépression, anxiété). Lors d'une seconde étape, les données brutes obtenues aux 20 échelles du MCMI sont transformées en résultats basaux, conversion qui repose sur des données relatives à la prévalence de chacun des troubles mentaux évalués. Finalement. les résultats basaux sont interprétés à partir de deux seuils de discrimination. Ainsi, un résultat basal supérieur à 74 et inférieur à 85 correspond à la présence de caractéristiques propres à un trouble mental, alors qu'un résultat basal supérieur a 84 représente une présence marquée de telles caractéristiques.

\section{RÉSULTATS}

À la figure 1, on retrouve les taux basaux moyens des violeurs peu violents physiquement ainsi que ceux des violeurs très violents physiquement, et ce pour les 11 échelles du MCMI qui concernent les troubles de la personnalité. En ce qui a trait aux violeurs peu violents physiquement. ils ont un taux basal moyen significativement plus élevé que les violeurs très violents physiquement aux échelles des troubles de la personnalité évitante, $\mathrm{F}(1,47)=12,7, p<.001$ et état-limite, $\mathrm{F}(1,47)=5.9, p<.05$. De plus, ils ont présenté un taux basal moyen marginalement plus élevé à l'échelle du trouble de la personnalité dépendante, $F(1.47)=3,6, p=.06$. Quant aux violeurs plus violents physiquement, ils ont un taux basal moyen significativement plus élevé que les violeurs moins violents physiquement aux échelles des troubles de la personnalité antisociale, $\mathrm{F}(1,47)=9,9, p<.01$, narcissique, $\mathrm{F}(1,47)=12,3, p<.01$, histrionique, $\mathrm{F}(1,47)=6,9, p<.05$, et paranoïde, $\mathrm{F}(1,47)=4,2, p<.05$.

Au tableau 1, on retrouve les résultats basaux supérieurs aux deux seuils de discrimination. soit 75 et 85 . Tout comme pour les taux basaux moyens, ces fréquences diffèrent selon le type de violeur. Ainsi, pour le seuil de 75 , les violeurs peu violents physiquement ont obtenu une fréquence de résultats basaux significatifs plus élevé que les violeurs très violents physiquement à l'échelle du trouble de la personnalité évitante, $\chi^{2}(1)=6,4, p<.01$. Quant aux violeurs très violents physiquement, ils ont obtenu des fréquences de résultats basaux significatifs plus élevées que les violeurs peu violents physiquement aux echelles des troubles de la personnalité antisociale $\chi^{2}(1)=11,6, p<.01$. paranoïde, $\chi^{2}(1)=6.6$, $p<.01$ et narcissique $\chi^{2}(1)=4,5, p<.05$. En ce qui a trait aux fréquences 


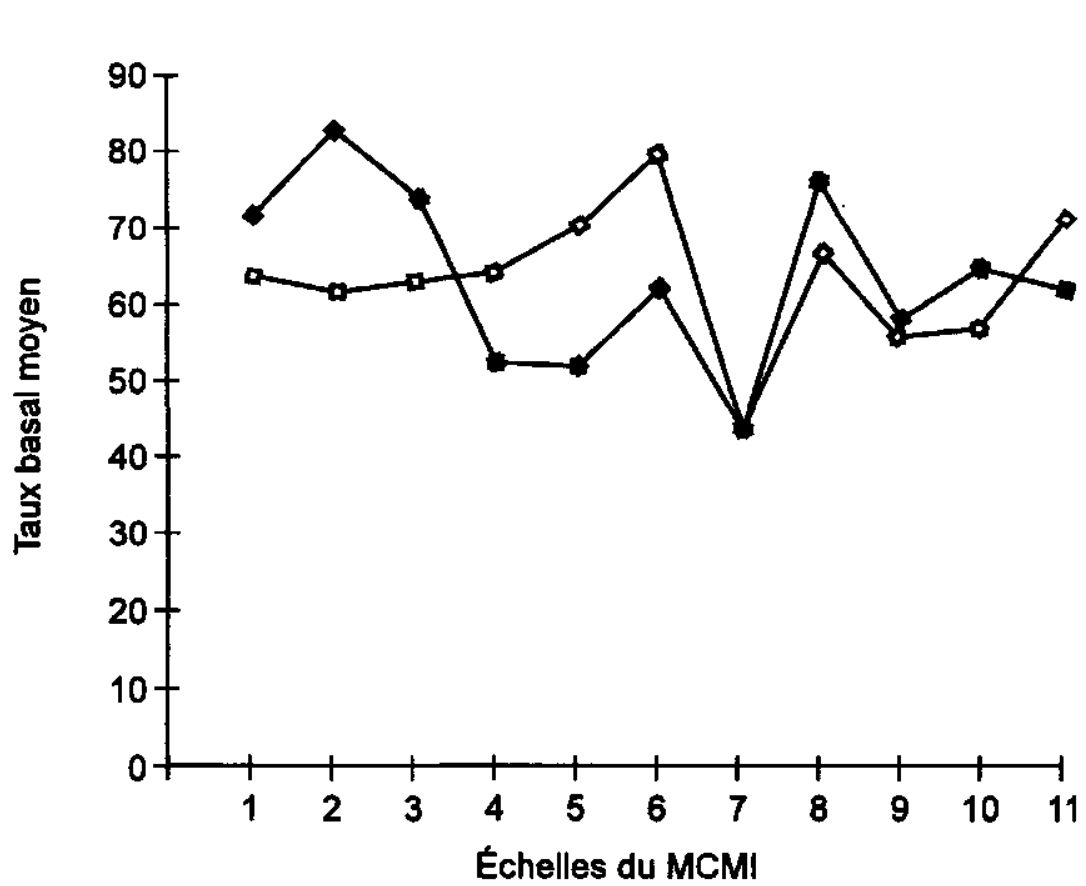

Figure 1. Taux basaux moyens au MCMI de violeurs peu violents physiquement et de violeurs très violents physiquement. Les nombres en abscisse correspondent aux onze troubles de la personnalité suivants : schizoïde (1), évitant (2), dépendant (3), histrionique (4), narcissique (5), antisociale (6), obsessionnelle-compulsive (7), passive-agressive (8), schizotypique (9), état-limite (10), paranoïaque (11). 
des résultats basaux supérieurs au seuil de discrimination de 85 , les différences significatives entre les deux types de violeurs sont les mêmes que celles obtenues au seuil de 75 .

En ce qui a trait aux violeurs peu violents physiquement, 30 d'entre eux ont présenté au moins un résultat basal supérieur au seuil de 74 pour une des échelles évaluant les troubles de la personnalité $(\bar{X}=3,6)$. Des pourcentages élevés $(>60 \%)$ de résultats basaux supérieurs au seuil de 74 furent obtenus pour les troubles de la personnalité évitante, dépendante, passive-agressive et schizoïde (voir tableau 1). De plus, 22 des 31 violeurs peu violents physiquement ont obtenu au moins un résultat basal supérieur au seuil de 84 pour une des échelles touchant les troubles de la personnalité $(\overline{\mathrm{X}}=1,7)$.

\begin{tabular}{|c|c|c|c|c|c|c|c|c|}
\hline \multirow[b]{3}{*}{ Échelle } & \multicolumn{4}{|c|}{ TABLEAU 1} & saux & ignifi & & \\
\hline & \multicolumn{4}{|c|}{$\begin{array}{l}\text { Peu violent physiquement } \\
\qquad \mathbf{N}=31\end{array}$} & \multicolumn{4}{|c|}{$\begin{array}{l}\text { Très violent physiquemen } \\
\qquad N=18\end{array}$} \\
\hline & \multicolumn{2}{|c|}{$75+$} & \multicolumn{2}{|c|}{$85+$} & \multicolumn{2}{|c|}{$75+$} & \multicolumn{2}{|c|}{$85+$} \\
\hline Schizoide & 61,2 & (19) & 22,5 & (7) & 44,4 & (8) & 27,8 & (5) \\
\hline Évitant & 77,4 & (24) & 51,6 & (16) & 38,9 & $(7)$ & 22,5 & (4) \\
\hline Dépendant & 74,2 & (23) & 38,7 & (12) & 55,5 & (10) & 33,3 & (6) \\
\hline Histrionique & 0 & $(0)$ & 0 & $(0)$ & 11,1 & (2) & 5,6 & (1) \\
\hline Narcissique & 9,6 & (3) & 3,2 & (1) & 38,8 & (7) & 27,8 & (5) \\
\hline Antisociale & 29,0 & (9) & 6,4 & (2) & 83,3 & (15) & 33,3 & (6) \\
\hline $\begin{array}{l}\text { Obsessionnelle- } \\
\text { compulsive }\end{array}$ & 0 & $(0)$ & 0 & $(0)$ & 0 & $(0)$ & 0 & $(0)$ \\
\hline $\begin{array}{l}\text { Passive- } \\
\text { agressive }\end{array}$ & 64,5 & (20) & 41,9 & (13) & 38,9 & (7) & 27,8 & (5) \\
\hline Schizotypique & 3,2 & (1) & 0 & (0) & 0 & $(0)$ & 0 & $(0)$ \\
\hline $\begin{array}{l}\text { État-limite } \\
\text { (Borderline) }\end{array}$ & 29,1 & (9) & 3,2 & (1) & 11,1 & (2) & 0 & $(0)$ \\
\hline Paranoïaque & 16,0 & (5) & 3,2 & (1) & 55,5 & (10) & 33,3 & (6) \\
\hline
\end{tabular}

Pour ce qui est des 18 violeurs très violents physiquement, ils ont tous présenté au moins un résultat basal supérieur au seuil de 74 pour une des échelles évaluant les troubles de la personnalité $(\bar{X}=3,7)$. Un pourcentage élevé de résultat basal supérieur au seuil de 74 fut obtenu pour le trouble de la personnalité antisociale (voir tableau I). En outre, 15 des 18 violeurs très violents physiquement ont présenté au moins un résultat basal supérieur au seuil de 84 pour une des échelles touchant les troubles de la personnalité $(\overline{\mathrm{X}}=2,1)$. 


\section{INTERPRÉTATION DES RÉSULTATS}

La plupart des violeurs de notre échantillon (98\%) ont présenté au moins un résultat basal supérieur au seuil de 74 pour une des échelles du MCMI évaluant les troubles de la personnalité. Ces résultats démontrent que l'incidence des troubles de la personnalité est très élevée chéz les violeurs. Ils concordent avec ceux obtenus par Langevin et al. (1988), lesquels indiquent que $90.2 \%$ des sujets de leur échantillon. composé de violeurs et de pédophiles, ont obtenu au moins un résultat basal supérieur au seuil de 74 pour une des échelles du MCMI, qui évalue les troubles de la personnalité.

Dans le cas des violeurs peu violents physiquement, des pourcentages élevés de résultats basaux significatifs furent obtenus pour les troubles de la personnalité évitante, dépendante, passive-agressive et schizoïde. Pour les violeurs très violents physiquement, un pourcentage élevé fut enregistré pour le trouble de la personnalité antisociale. Ces donnés, concernant deux types de violeurs, sont similaires à celles obtenues par Bard et Knight (1987) auprès d'un échantillon composé de violeurs et de pédophiles. En effet, leur premier type d'agresseurs sexuels. peu violents physiquement lors du délit, se caractérise par des élévations significatives aux échelles évaluant les troubles de la personnalité évitante. dépendante et schizoïde. Ces troubles de la personnalité sont les mêmes que ceux identifiés dans notre groupe de violeurs peu violents physiquement. Quant au second type d'agresseurs sexuels, identifié par Bard et Knight, il se distingue par un niveau de violence physique important lors du délit et des élévations significatives aux échelles du MCMI qui concernent les troubles de la personnalité antisociale, narcissique et histrionique. Ce groupe d'agresseurs sexuels, composé à $88 \%$ de violeurs, présente un profil de troubles de la personnalité similaire à celui obtenu auprès de notre échantillon de violeurs très violents physiquement. Dans là même veine, Rice, Harris et Quinsey (1990) ont établi un lien entre un niveau élevé de violence physique lors d'un viol et des résultats élevés à l'échelle de psychopathie de Hare. Ainsi, malgré l'hétérogénéité de l'échantillon d'agresseurs sexuels de Bard et Kight, leurs resultats sont équivalents à ceux obtenus auprès d'un échantillon constitué uniquement de violeurs. Cette équivalence suggère que dans l'évaluation des troubles de la personnalité, le niveau de violence physique exercée lors du délit est un facteur permettant d'identifier les types d'agresseurs sexuels, ce qui n'est pas le cas avec l'âge des victimes. Cela fut également observé dans les études réalisées avec le MMPI (Armentrout et Hauer, 1978; Hall, Graham et Shepherd, 1991 ; Kalichman, 1991 : Panton, 1978) et avec la phallométrie (Proulx, Aubut. McKibben et Côté, 1994). De manière différente, l'analyse de variance effectuée confirme que le niveau de 
violence physique lors du délit est un facteur qui permet de discriminer les divers types de violeurs.

Nos résultats indiquent que la quasi-totalité des violeurs présentent des troubles de la personnalité et que selon la nature de ces troubles, ils ont commis des viols qui diffèrent par leur niveau de violence physique. Mais quelle est la signification de cette association entre troubles de la personnalité et scénario du délit? Afin de répondre à cette question, une avenue qui nous semble pertinente à explorer serait la conception des troubles de la personnalité développée par Millon (1981). Selon cet auteur, un individu qui présente un trouble de la personnalité se distingue par un mode relationnel habituel, inapproprié et rigide, qui engendre des conflits interpersonnels ou une détresse intérieure. Ainsi, si l'on considère que le viol constitue une forme de rapport interpersonnel et que la nature du trouble de la personnalité détermine les caractéristiques de ce type de rapport, il est plausible que la nature du trouble de la personnalité va également déterminer le scénario du viol dans ses spécificités intra-individuelle et interindividuelle. Ainsi, le viol serait un prolongement dans le champ de la sexualité du mode relationnel habituel associé à un trouble de la personnalité, et ce dans ses dimensions cognitives, affectives et comportementales. Cette conception ne suppose pas que la présence d'un trouble de la personnalité soit une condition suffisante pour qu'un individu commette un viol. Cependant, il semble que la présence d'un tel trouble soit une condition nécessaire à l'émergence et à la structuration d'un viol.

À titre d'exemple, prenons le cas d'un homme présentant un trouble de la personnalité antisociale. Celui-ci considère, lors de ses rapports avec autrui, qu'il ne reçoit pas sa juste part, qu'on est injuste à son égard. Il en résulte de la colère, exprimée par des actes de violence đivers (Beck et Freeman,1990). Dans le champ plus particulier de ses rapports avec les femmes, il se perçoit également comme une victime d'injustice. Ces perceptions suscitent chez lui une colère contre les femmes, laquelle est exprimée lors d'un viol comportant un niveau élevé de violence physique. Ainsi, pour un individu présentant un trouble de la personnalité antisociale, les caractéristiques cognitives, affectives et comportementales associées à son mode relationnel habituel se retrouvent dans des comportements coercitifs dont la nature sexuelle ou non sexuelle est fonction de son histoire d'apprentissage.

Prenons un autre exemple, soit le cas d'un homme présentant un trouble de la personnalité évitante et qui aurait perpétré un viol comportant peu de violence physique. Celui-ci se croit incompétent et inférieur à autrui dans ses rapports interpersonnels. Il en résulte un sentiment d'humiliation et des comportements visant à rabaisser une personne qu'il considère lui être inférieure. Ces comportements ont pour but de diminuer son propre 
sentiment d'humiliation tout en restaurant de façon illusoire son estime personnelle. Dans ses interactions avec les femmes également, il se perçoit comme inférieur et il se sent humilié. Il a alors recours au viol pour humilier une femme. Il est à noter que lors d'une évaluation phallométrique. ce violeur a présenté une préférence sexuelle pour des stimuli de viol comportant de la sexualité et de l'humiliation (Proulx. Aubut, McKibben et Côtế, sous presse). Ainsi dans les deux exemples présentés. les scénarios de viol constituent des prolongements directs, dans la sphère sexuelle, des scénarios relationnels spécifiques associés à des troubles de la personnalité.

Ces liens que nous avons établis entre les troubles de la personnalité et le viol permettent d'aborder sous un angle différent des questions laissées en suspens dans les théories récentes du viol (Hall et al.. 1991; Malamuth et al., 1993: Marshall et Barbaree, 1990). Tout d'abord, dans chacune de ces théories du viol, un seul type de personnalité (antisociale ou narcissique) est pris en considération comme facteur causal. Dans la conception du viol que nous suggérons, plusieurs types de personnalité peuvent être impliqués dans les processus qui concourent à la perpétration d'un viol, ce qui concorde avec les résultats d'études psychométriques. phallométriques et typologiques. $\grave{A}$ un autre niveau. les théories récentes du viol proposent un modèle universel du viol qui est incompatible avec la diversité phénoménologique des types de viols perpétrés. Quant à notre conception du viol. elle explique cette diversité phénoménologique, laquelle serait attribuable principalement à une autre diversité. soit celle des troubles de la personnalité présents chez les auteurs de ces viols. Sur ce point. notre conception du viol offre également des éléments de compréhension. En effet, le mode relationnel, caractéristique de chaque trouble de la personnalité, présente une structure interactive spécifique composée de facteurs cognitifs, affectifs et comportementaux (Beck et Freeman, 1990). Or ces modes relationnels, selon notre conception, constituent les matrices desquelles découlent des scénarios spécifiques de viol. En conséquence. il semble probable que ces scénarios de viol présentent les mêmes structures interactives que les modes relationnels dont ils originent. Ainsi, la conception du viol qui découle des résultats de la présente étude offre une perspective qui diffère de celles des théories récentes du viol, et ce même si elle est dans le sillage de celles-ci. Cette conception offre également des similitudes avec les théories phénoménologiques et psychanalytiques du viol.

Selon les tenants des théories phénoménologiques du viol, cet acte est pseudo-sexuel et répond à des impératifs affectifs. Ainsi. pour un type de violeurs, l'expression de la colère constituerait la motivation primaire du viol (Cohen, Garafalo. Boucher et Seghorn, 1971; Cohen, Seghorn et Calmas, 1969 ; Gerhard. Gagnon, Pomeroy et Christenson, 1965; Groth et Birnbaum, 1979; Groth, Burgess et Holmstrom, 1977: Knight et Prentky. 1990). Pour un autre type de violeurs. cet acte viserait à restaurer temporai- 
rement un sentiment de pouvoir, de contrôle. qui compenserait un sentiment d'humiliation relatif à des événements à caractère sexuel et non sexuel (Cohen et al., 1971; Cohen et al., 1969; Groth et Birnbaum, 1979; Groth et al. 1977; Knopp, 1962). Cette conception du viol. comme celle que nous avons suggérée, en souligne la fonction affective et sa relation avec les caractéristiques de la personnalité du violeur. De plus, les deux principaux types de violeurs mentionnés dans les théories phénoménologiques du viol correspondent à ceux identifiés dans la présente étude, tant sur le plan des caractéristiques de la personnalité que sur celui du scénario délictuel.

Les théories psychanalytiques du viol présentent également des similitudes avec la conception du viol suggérée dans cet article. Ainsi, dans celles-ci, les types de viols. comme comportements déviants, seraient en relation avec des modes spécifiques de relation objectale (Demoulin. Donnay-Richelle et Timsit. 1973; Schorsch, Galedary. Haag, Hauck et Lohse. 1990; Van Gijseghem, 1988). À titre d'exemple, selon Schorch et al. (1990), les symptômes déviants auraient des fonctions diverses tel qu'exprimer de la rage ou compenser une masculinité incertaine. Ainsi. dans les théories psychanalytiques du viol, tout comme dans notre propre conception, on retrouve plusieurs types de violeurs, lesquels présentent des profils de personnalité qui sont en relation directe avec le scénario du délit.

Ainsi, la conception du viol proposée dans cet article permet un rapprochement entre d'une part les théories psychanalytiques et phénoménologiques, dans lesquelles l'accent est mis sur la fonction affective du délit, et d'autre part les théories cognitivo-comportementales, dans lesquelles la structure interactive des facteurs étiologiques est négligée au profit du lien statistique établi entre ceux-ci et cette varíable dépendante qu'est le viol. Toutefois. la justesse des interprétations faites sur la base de nos résultats requiert des confirmations empiriques. Tout d'abord, il serait nécessaire d'effectuer des études dans lesquelles seraient mis en relation des troubles de la personnalité et les préférences sexuelles telles qu'évaluées par la phallométrie. De telles études permettraient de vérifier si les scénarios de fantasmes de viol, tout comme les scénarios de délits, présentent les mêmes structures interactives que ceux des troubles de la personnalité desquels on suppose qu'ils découlent. Des études qualitatives devraient aussi être réalisées, afin de vérifier si les caractéristiques cognitives, affectives et comportementales des scénarios fantasmatiques et délictuels correspondent à des scénarios relationnels spécitiques lors d'interactions actuelles. Par-delà l'intérêt théorique de la conception du viol proposée ici, celle-ci comporte également des implications sur le plan clinique. 


\section{IMPLICATIONS CLINIQUES}

Les liens existant entre les troubles de personnalité et le viol peuvent être considérés sous trois aspects. Premièrement, il y a les liens de nature étiologique. c'est-à-dire les troubles de personnalité en tant que facteurs causals du viol. Cet aspect a été discuté dans la première partie de cet article. Deuxièmement, il y a les liens de modulation du délit. Cet aspect a été traité partiellement dans la partie précédente de cet article. En effet. notre étude a permis de démontrer qu'il existait un lien entre les troubles de personnalité et le niveau de violence lors du délit. Plusieurs autres aspects pourraient également être investigués. Nous n'en retiendrons ici que deux, à savoir le choix de la victime et la récidive. Ainsi, les victimes peuvent être choisies totalement au hasard, en fonction d'attributs extérieurs ou encore en fonction d'attributs intérieurs. Les personnalités présentant un niveau élevé d'impulsivité, telle la personnalité antisociale, peuvent avoir tendance à choisir leur victime au hasard ou encore en fonction de circonstances environnementales qui pourraient leur assurer l'impunité. Les personnalités de type narcissique auront tendance pour leur part à choisir les victimes en fonction d'attributs extérieurs. Ainsi, on peut rapporter le cas d'un violeur qui choisissait des victimes qui portaient des manteaux de fourrure ou conduisaient des voitures luxueuses. Ce violeur ne pouvait agresser que des victimes qui lui paraissaient d'un niveau socio-économique supérieur. Le choix des victimes était donc clairement motivé par des éléments d'ordre narcissique. Les personnalités dépendantes choisiront pour leur part d'agresser des victimes en fonction de certains attributs intérieurs, c'est-à-dire en fonction de liens réels ou imaginaires avec la victime. Par exemple, certains violeurs vont agresser surtout des femmes qu'ils connaissent et vis-à-vis lesquels ils ont créć un lien de dépendance. L'agression surviendra dans un contexte de séparation et servira vainement à maintenir la relation qui s'effrite.

En ce qui a trait à la récidive, seule la personnalité antisociale a été clairement identifiée comme étant un facteur de risque accru, et ce pour une variété importante de délits, quelle qu'en soit la nature (Rice. Harris et Quinsey, 1990). Il y a certainement une surreprésentation des études sur la personnalité antisociale dans la littérature scientifique, probablement parce qu'elle est mieux définie que d'autres types de personnalité et que certains instruments de mesure objectifs ont été mis sur pied pour l'étudier (ex. : échelle de psychopathie de Hare). Il y aurait certainement lieu d'étudier de manière plus objective les rapports existants entre la récidive et les différents types de personnalité.

Troisièmement. il y a les liens entre les troubles de la personnalité et le traitement. Ces liens seront considérés sous quatre aspects, soit l'engagement dans le traitement. le processus thérapeutique, la fin du traitement et 
les ajustements post-thérapeutiques. Il n'est pas question ici de couvrir pour chacune de ces étapes l'ensemble des problèmes occasionnés par le fait d'être porteur d'un trouble de personnalité. Il s'agit plutôt d'illustrer comment ces étapes seront modulées en fonction d'un trouble de personnalité spécifique.

\section{ENGAGEMENT DANS LE TRAITEMENT}

L'une des grandes caractéristiques des personnes atteintes de troubles de la personnalité réside dans l'attribution du blâme à autrui. En effet, pour la plupart, ils attribuent la responsabilité de ce qui leur arrive à autrui : leurs parents, leur conjoint. les instances légales, la société en général. Souvent, ils n'ont pas l'impression d'être maîtres de leur destinée et ils se perçoivent comme contrôlés par des facteurs extérieurs à eux-mêmes. En outre, l'expérience subjective de la souffrance est souvent diffuse, mal cernée et encore une fois attribuée à des responsables extérieurs. L'ensemble de ces phénomènes agira évidemment sur l'engagement dans le traitement. En effet, les violeurs atteints de troubles de la personnalité ne se perçoivent pas comme responsables de leur vie en général, ni de leurs actions, et ils parviennent mal à cerner leurs difficultés réelles. Au lieu de se percevoir comme des agresseurs, ils ont plutôt tendance à se percevoir comme des victimes. Dans un tel contexte, on comprendra facilement que la motivation au changement est plutôt aléatoire. L'engagement dans le traitement sera souvent favorisé par un agent extérieur, après un arrêt d'agir, suite à une intervention légale. S'ils consultent d'eux-mêmes, en dehors de tout contexte légal, les violeurs le font en général en rapport avec des problèmes autres que sexuel, soit des difficultés conjugales. l'alcoolisme ou la toxicomanie.

L'engagement dans le traitement sera fortement modulé par le type de troubles de la personnalité. Ainsi. les violeurs présentant des troubles de la personnalité du groupe dramatique tels les antisociaux ou les narcissiques peuvent avoir au départ une attitude plutôt séductrice. Au contraire, ceux présentant des troubles de la personnalité du groupe excentrique tels les schizotypique auront tendance à présenter pour leur part des scénarios sexuels bizarres hors du commun laissant le thérapeute parfois perplexe. La sexualité et l'agressivité sont souvent intriquées de manière diffuse dans leur scénario. La demande d'aide sera souvent également difficile à cerner. Enfin, les violeurs manifestant des troubles de la personnalité du groupe anxieux tels les passifs-agressifs s'engageront dans le traitement en attendant du thérapeute qu'il fasse tout le travail. Ils disent s'en remettre entièrement au thérapeute, mais dans les faits, leur résistance passive au changement se manifestera asse\% rapidement. 


\section{PROCESSUS THÉRAPEUTIQUE}

En cours de route, une série d'attitudes et de comportements sera mise en évidence. Encore une fois. l'approche par regroupement de types de troubles de la personnalité tels que définis dans le DSM-III-R semble être utile. En effet, les violeurs présentant des troubles de la personnalité du groupe dramatique tels les états-limites. les narcissiques ou les antisociaux céderont assez rapidement le pas à la séduction pour s'engager dans un processus de confrontation et de contrôle de la thérapie et du thérapeute. C'est la dimension de rage et de confrontation directe et ouverte qui caractérise ce groupe. Les violeurs présentant des troubles de la personnalité du groupe excentrique présenteront d'autres niveaux de problèmes. En effet. ils ont tendance à être présents aux différentes modalités thérapeutiques qui leur sont offertes. mais ils ne semblent pas être a leur place quelle que soit la modalité offerte. Ils continuent à laisser le thérapeute perplexe. Ils continuent à vivre dans un monde imaginaire et semblent peu perméables à la réalité d'autrui. Enfin, ceux manifestant des troubles de la personnalité du groupe anxieux manifesteront leur résistance au traitement sur un mode passif. Ils seront rarement en confrontation directe, comme c'est le cas des états-limites, par exemple. Ils confrontent plutôt le therapeute par leur silence, leurs oublis, leur incompréhension ou leur absence d'adhésion aux consignes thérapeutiques. Ainsi, dans les groupes d'habiletés sociales, ils ne feront pas les exercices requis en dehors des séances.

\section{FIN DE TRAITEMENT}

Pour les violeurs chez qui l'on retrouve des troubles de la personnalité du groupe dramatique, la fin du traitement sera en général plutôt abrupte, associé à un passage à l'acte soit en rapport avec la thérapie ou encore à un passage à l'acte dans leur vie. Ainsi, ils cesseront abruptement de venir au rendez-vous et refuseront les rappels pour qu'une mise au point soit effectuée. Ce type de fin de traitement se situe tout à fait dans la lignée des troubles de personnalité état-limite pour qui la vie est une suite d'engagements et de ruptures. Les violeurs présentant des troubles de la personnalité du groupe excentrique mettront fin au traitement en général lorsqu'ils adhéreront à une nouvelle idéologie mystique, pseudo-mystique ou ésotérique. Souvent, ils s'inséreront dans un groupe ésotérique qui correspond en apparence un peu plus à leurs convictions marginales. Ils vont trouver au sein de ces groupes. la plupart du temps, un certain sentiment d'appartenance, une certaine insertion sociale et aussi un cadre qui jugule leurs pulsions qu'ils parviennent plus ou moins bien à métaboliser. En effet. la plupart des groupes auxquels ils adhèrent ont des règles très strictes en ce qui a trait à la sexualité. Souvent, il y aura des allers et des retours entre les groupes 
ésotériques et le traitement. Enfin, ceux présentant des troubles de la personnalité du groupe anxieux continueront dans la même veine de passivité. Ce sont en général les thérapeutes qui mettront fin au traitement, puisqu'ils vivront un sentiment d'impuissance face à leur incapacité de percer la carapace défensive des anxieux. Ce type de violeurs se sentira encore une fois victimisé et ne comprendra pas toute l'agressivité qu'ils parviennent fort bien à mobiliser chez les thérapeutes.

\section{AJUSTEMENTS POST-THÉRAPEUTIQUES}

Tous les programmes de traitement pour les violeurs visent bien sûr au départ à ce que ceux-ci acquièrent une meilleure gestion pulsionnelle, c'està-dire qu'ils ne récidivent pas. La prévention de la récidive ne saurait être le seul objectif d'un programme de traitement, puisque la qualité de vie des violeurs aura un impact important sur leur récidive. Par qualité de vie, nous entendons la capacité d'acquérir une meilleure gestion dans la relation à autrui et une capacité d'acquérir une meilleure gestion dans la relation à soi-même, c'est-à-dire une meilleure estime de soi. En effet, les violeurs qui seraient capables d'établir des relations affectives stables, satisfaisantes et gratifiantes présenteront certainement moins de risques de récidive. À l'inverse, ceux qui sont incapables d'établir des relations satisfaisantes avec des femmes en particulier et qui maintiennent des modes relationnels basés sur le contrôle. la domination ou l'humiliation sont certainement plus à risque. Il s’agit là en soi de banalités, mais elles prennent leur sens lorsqu'il faut élaborer des programmes de traitement qui tiennent compte de manière spécifique de ces dimensions. Les violeurs qui ont une estime d'eux-mêmes trop faible ou encore trop élevée demeurent aussi plus à risque. En général. les programmes de traitement pour les agresseurs sexuels s'adressent à ces dimensions par l'entraînement aux habiletés sociales ou par d'autres modalités. Il importe par ailleurs qu'un suivi serré soit accordé aux violeurs en externe afin de s'assurer du maintien des acquis tant sur le plan de la gestion pulsionnelle, que de la gestion de la relation à autrui et de la gestion de la relation à soi. L'entraînement à cette gestion dans le cadre d'un laboratoire ou d'une thérapie ne prend son sens que dans la mesure où il y a validation des acquis dans la réalité et ce sur une période de temps assez. longue.

Dans la première partie de cet article, nous avons démontré qu'il était possible d'établir une relation entre les troubles de personnalité et une variable importante lors d'un délit chez les violeurs, soit le niveau de violence. Dans la deuxième partie, nous avons ouvert d'autres avenues pour explorer les liens entre trouble de personnalité et viol. Ces avenues ont été ouvertes à titre indicatif seulement, sans être exhaustives. Il nous apparaît clair que la recherche scientifique doit s'ouvrir aux préoccupations 
cliniques et vice versa. Les programmes de traitement ne sauraient évoluer sans un apport rigoureux sur le plan scientifique. À l'inverse. la recherche est bonifiée lorsqu'elle suit son objet d'étude dans toutes les phases de sa réalité et de sa complexité.

\section{BIBLIOGRAPHIE}

AMERICAN PSYCHIATRIC ASSOCIATION (1987), Diagnostic and Statistical Manual of Mental Disorders ( $3^{c}$ edition revisée), Washington, DC.

ANDERSON, W. P., KUNCE, J. T. et RICH, B. (1979), «Sex Offenders : Three Personality Types", Journal of Clinical Psychology, vol. 55, pp. 671-676.

ARMENTROUT, J. A., et HAUER, A. L. (1978), « MMPI of Rapists of Adults, Rapists of Children and Non-Rapist Sex Offenders n, Journal of Clinical Psychology, vol. 34, pp. 330-332.

AVERY-CLARK, C. A. et LAWS, D. R. (1984), «Differential Erection Response Pattems of Sexual Child Abusers to Stimuli Describing Activities with Children », Behavior Therapy, vol. 15, pp. 71-83.

BARD, L. A. et KNIGHT, R. A. (1987), "Sex Offender Subtyping and the MCMI», texte présenté à la Conjerence on the Millon Clinical Inventory, Miami, Floride.

BARLOW, D.H. et ABEL, G. G. (1976), « Sexual Deviation", in W. E. CRAIGHEAD, A. E. KAZDIN et N. J. MALONEY (dir.), Behavior Modification: Principles, Issues and Applications, Boston, Houghton et Mifflin, pp. 341-360.

BECK, A.T. et FREEMAN, A. (1990), Cognitive Therapy of Personality Disorders, New York, Guilford.

BOND, I. K. et EVANS, D. R. (1967), « Avoidance Therapy : Its Use in Two Cases of Underwear Fetishism», Canadian Medical Association Journal, vol. 96, pp. 1160-1162.

BUTCHER, J. et TELLEGEN, A. (1978), «Common Methodological Problems in MMPI Research», Journal of Consuling and Clinical Psychology, vol. 46, pp. $620-628$.

COHEN, M. L., SEGHORN, T. et CALMAS, W. (1969), "Sociometric Study of Sex Offenders », Journal of Abnormal Psychology, vol. 74, pp. 249-255.

COHEN, M. L., GAROFALO, R. F., BOUCHER, R. et SEGHORN, T. (1971), « The Psychology of Rapists ", Seminars in Psvchiatry, vol. 3, pp. 307.327.

D'ELIA, A. (1988), A Study of Personatity Patterns in Homosexual and Heterosexual Pedophiles, thèse inédite, universitê McGill, Montréal, Québec.

DEMOULIN, C., DONNAY-RICHELLE, J. et TIMSIT, M. (1973), «Siructure perverse et perversions sexuelles $n$, Acta psychiatrica belgica, vol. 73, pp. 725-746.

GEBHARD, P. H., GAGNON, J. H., POMEROY, W. B. et CHRISTENSON, C. V. (1965), Sex Offenders : An Analysis of Types, New York, Harper \& Row.

Groth, A. N. el BIRnBaUM, H. J. (1979), Men Who Rape, New York, Plenum.

GROTH, A. N., BURGESS, A. W. et HOLMSTROM, L. L. (1977), « Rape : Power, Anger and Sexuality", Americtin tournat of Psychiatry, vol. 134, pp. 1239-1243. 
HALL, G. C. N., GRAHAM, J. R. et SHEPHERD, J. B. (1991), "Three Methods of Developing MMPI Taxonomies of Sexual Offenders", Journal of Personality Assessment, vol. 56, pp. 2-13.

HALL, G. C. N. et HIRSCHMAN, R. (1991), "Towards a Theory of Sexual Aggression : A Quadripartite Model », Journal of Consulting and Clinical Psychology, vol. 59, pp. 662-669.

KALICHMAN, S. (1990), "Alfective and Personality Characteristics of MMPI Profils Subgroups of Incarcerated Rapists». Archives of Sexual Behavior, vol. 19, pp. 443-459.

KALICHMAN, S. (1991), «Psychopathology and Personality Characteristic of Abnormal Sexual Offenders as a Function of Victim Age $*$, Archives of Sexual Behavior, vol. 20, pp. 187-197.

KALICHMAN, S. C., SZYMANOWSKI, D., MCKEE, J., TAYLOR, J. et CRAIG, M. (1989), «Cluster Analytically Derived MMPI Profils Subgroups of Incarcerated Adult Rapists ", Journal of Clinical Psychology, vol. 45, pp. 149-155.

KARACAN, I., WILLIAMS, R. L., GUERRERO, M. W., SCALES, P. J., THORNBY, J. I. et HURSCH, T. (1974), " Norturnal Penile Tumescence and Sleep of Convicted Rapists and Other Prisoners ", Archives of Sexual Behavior, vol. 3, pp. 19-26.

KNIGHT, R. A. et PRENTKY, R. A. (1990), «Classifying Sexual Offenders: The Development and Corroboration of Taxonomic Models $n$, in W. L. Marshall, D. R. Laws et H. E. Barbaree (dir.), Handbook of Sexual Assault: Issues, Theories and Treatment of The Offender, New York, Plenum, pp. 23-52.

KNIGHT, R. A., ROSENBERG, R. et SCHNEIDER, B. (1985), "Classification of Sexual Offenders : Perspectives, Methods, and Validation», in A. Burgess (dir.), Rupe and Sexual Assault: A Research Handbook, New York, Garland, pp. 222293.

KOPP, S. B. (1962), «The Character Structure of Sex Offenders », American Journal of Psychothercipy, vol. 16, pp. 64-70.

LANGEVIN, R., LANG, R., REYNOLDS, R., WRIGHT, P., GARRELS, D., MARCHESE, V., HANDY, L., PUGH, G. et FRENZEL, R. (1988), « Personality and Sexual Anomalies : An Examination of the Millon Clinical Mutiaxial Invertory ", Annals of sex Research, vol. 1, pp. 13-32.

LEVIN, S. M. et STAVA, L. (1987), "Personality, Characteristics of Sex Offenders : A Review n, Archives of Sexual Behavior, vol. 16, pp. 57-79.

MALAMUTH, N. M. (1986), «Predictors of Naturalistic Sexual Aggression ", Journal of Personality and Social Psychology, vol. 50, pp. 953-962.

MALAMUTH, N. M., HEAVEY, C. L. ct LINZ, D. (1993), « Predicting Men's Antisocial Behavior Against Women : The Interaction Model of Sexual Aggression», in G. C. N. Hall, R. Hirschman, J. R. Graham et M. C. Zaragoza (dir.), Sexual Aggression: Issues in Etiology, Assessment and Treatment, Washington, Taylor \& Francis, pp. 63-97.

MALAMUTH, N. M., SOCKLOSKIE, R., KOSS, M. P. et TANAKA, J. (1991), « The Characteristics of Aggressors Against Women : Testing a Model Using a Natural Sample of College Students», Journal of Consulting and Clinical Psychology, vol. 59, pp. 670-681.

MARSHALL, W. L. et BARBAREE, H. E. (1990), "An Integrated Theory of the Etiology of Sexual Offending », in W. L. Marshall, D.R. Laws et H. E. Barbaree 
(dir.), Handbook of Sexual Assault: Issues, Theories and Treatment of the Offender, New York, Plenum, pp. 23-52.

MARSHALL, W. L., EARLS, C. M., SEGAL, Z., et DARKE, J. L. (1983), « A Behavioral Program for the Assessment and Treatment of Sexual Aggressors», in K. D. Craig et R. J. McMahon (dir.), Advances In Clinical Behavior Therapy, New York, Brunner Mazel, pp. 148-174.

MILLON, T. (1981), Disorders of Personality : DSM-III, Axe II, New York, Wiley.

MILLON, T. (1983), Millon Clinical Multiaxial Inventory Manual, Minneapolis : Interpretative Scoring Systems.

PANTON, J. H. (1958), MMPI «Configurations Among Crime Classification Groups ", Journal of Clinical Psychology, vol. 14, pp. 305-308.

PANTON, J. H. (1978), «Personality Differences Appearing Between Rapists of Adults, Rapists of Children And Non-Violent Sexual Molesters of Female Children », Research Communications in Psychology, Psychiatry and Behavior, vol, 3, pp. 385-393.

PROULX, J., AUBUT, J., MCKIBBEN, A. et COTÉ, M. (1994), « Penile Responses of Rapists and Nonrapists to Rape Stimuli Involving Physical Violence or Humiliation », Archives of Sexual Behavior, vol. 23, 3, pp. 295-310.

QUINSEY, V. L., ARNOLD, L. S. et PRUESSE, M. G. (1980), "MMPI Profiles of Men Referred for a Pretrial Psychiatric Assessment as a Function of Offence Type », Journal of Clinical Psychology, vol. 36, pp. 410-417.

RICE, M. E., HARRIS, G. T. et QUINSEY, V. L. (1990), "A Follow-up of Rapists Assessed in a Maximum Security Psychiatric Facility ». Journal of Interpersonal Violence, vol. 5, pp. 435-448.

SCHORSCH, E., GALEDARY, G., HAAG, A., HAUCK, M. et LOSHE, H. (1990), Sex Offenders : Dynamics and Psychotherapeutic Strategies, Berlin, Springer-Verlag.

VAN GINSEGHEM, H, (1988), La Personnalité de l'abuseur sexuel: Typologie à partir de l'optique psychanalytique, Montréal, Édition du Méridien. 